\title{
Levels of processing: Qualitative differences or task-demand differences?
}

\author{
SHANNON DAWN MOESER \\ Memorial University of Newfoundland, St. John's, Newfoundland A1B 3X8, Canada
}

\begin{abstract}
In nearly all reported orienting task studies, the question is asked before the item or items are presented. This paper reports two experiments wherein the question was asked after the item was presented. These experiments found that an orthographic orienting task did not produce poorer retention than a semantic orienting task when (1) the orthographic task was prosented in such a way to ensure that the list items would be encoded as units and (2) the test was designed to eliminate the effect of encoding elaboration to positive-response orienting questions. It was concluded that the depth-of-processing effect was composed of two components. One of these is a task-demand component that affects the probability of encoding target items as identifiable units. The second component of processing depth is trace elaboration to positive-response questions. In most experiments, the two components combine to produce better memory performance for targets presented with semantic orienting questions. However, the two components can be examined independently of each other to determine the degree to which each contributes to a particular experimental effect.
\end{abstract}

There are a number of studies comparing the effects of different types of orienting tasks on the retention of verbal items (e.g., Craik \& Tulving, 1975; Hyde, 1973; Hyde \& Jenkins, 1969, 1973; Johnston \& Jenkins, 1971; Rosenberg \& Schiller, 1971; Schulman, 1971, 1974; Walsh \& Jenkins, 1973). In these studies, subjects are first given a task requiring them to analyze a list of stimulus items in terms of a particular characteristic and then are given an unexpected memory test on these items. Typically, the orienting tasks are classified as either semantic or nonsemantic in nature. Semantic tasks are those that require the subject to analyze the items in terms of their meaningful properties; nonsemantic tasks are those that draw the subject's attention to orthographic or phonological attributes of the items. Semantic orienting tasks have consistently produced better retention of the list items, both when recall tests are used and when recognition tests are used.

A levels-of-processing model has generally been used to interpret these findings. As initially proposed by Craik and Lockhart (1972), this model viewed the memory system as a linear sequence of analyzing operations, with structural analyses occurring prior to semantic analyses. The durability of the trace formed to represent the list item was assumed to be a function of the depth to which this item was processed. Items processed only in terms of their surface features would give rise to a very short-lived trace; items processed to a deeper semantic level would give rise to a more perm-

This research was supported by Grant A9638 from the Natural Sciences and Engineering Council of Canada. Reprint requests should be sent to Shannon Moeser, Department of Psychology, Memorial University of Newfoundland, St. John's, Newfoundland A1B 3X9, Canada. anent trace. In more recent versions of the model, the notion of a linear sequence of processing stages has been dropped, as has the notion of differences in trace durability (e.g., Fisher \& Craik, 1977; Jacoby \& Craik, 1978). Whereas Craik and Lockhart argued that the more deeply encoded traces decayed less rapidly than shallowly encoded traces, Jacoby and Craik suggested that deeper encodings were not more durable than shallow encodings but, instead, were more discriminable from other memory traces. This revised model still retains, however, the concept of different levels or domains of processing. That is, it assumes that there is a qualitative distinction between semantic and nonsemantic analyzing operations.

The notion of qualitative differences among levels has never been precisely explained or illustrated by proponents of the levels model. However, the term "qualitative differences" seems to suggest that a different type of code is used at each processing level. One way to formalize this view would be to assume that the verbal unit can be represented in a number of different ways-as a set of orthographic features, or a set of phonemic features, or a set of semantic features, and so on. In other words, we can assume that the physical features of the target item can be represented as a coherent knowledge unit without including in this representation any information about the item's semantic characteristics. This means that theoretically, at least, a trace formed as the result of an analysis of the target item could contain only orthographic features and yet possess all the information necessary to recreate a physical representation of the item.

The notion of a qualitative difference among encoding levels can be contrasted with a task-demand explanation such as that proposed by Morris, Bransford, and 
Franks (1977). The task-demand explanation assumes that an encoded event is always represented in a semantic memory code, whether this be information about the referential characteristics of the item or the surfacestructure characteristics. The orienting task will encourage specific types of semantic information to be represented. Thus, for example, the target word "cat" might be encoded in terms of its referential properties (the word represented the item "cat"), its orthographic properties (the word was presented in uppercase type), or its phonemic properties (the word rhymed with "bat"), but in all cases the representation is a semantic description of the event. When it is necessary to retrieve information about the target item, the encoded event must be recreated from the semantic code. Consequently, if only information about the orthographic properties of the word were encoded (i.e., that the word was presented in uppercase type), the encoder would not be able to identify the presented word on a test in which items were differentiated on the basis of their seman. tic characteristics.

In support of this task-demand explanation, Morris et al. (1977) found that recognition performance depended on the type of test given the subjects, rather than on the level of orienting task. Although semantic questions produced superior performance on a standard type of semantic recognition test, rhyming questions produced better performance on a rhyming recognition test. Similar findings have been reported by Fisher and Craik (1977). Fisher and Craik argue, however, that even when the appropriate retrieval test is used, performance in nonsemantic conditions is not equivalent to performance in semantic conditions. Consequently, they state that it is still necessary to retain the concept of different levels of processing to explain qualitative differences between semantic and nonsemantic orienting tasks.

The experiments in the present paper were designed to examine whether we could obtain evidence of qualitative differences in the codes used at the various levels of processing or whether the differences obtained in orienting task experiments were best explained in terms of a task-demand hypothesis. These experiments examined how verbal retention is affected when the target item is presented before the orienting question is asked. It is common practice in orienting task studies to present the target item after the orienting question. When the question-first order is used, however, the subjects are likely to organize their analysis of the presented items in terms of the question being asked (cf. Moeser, 1978). Consequently, subjects directed to attend to the orthographic features of a given item may encode only information about these orthographic features (i.e., that the word was presented in uppercase type) and fail to encode information about the referential properties of the word (i.e., that it represents the item "cat").

In all of the reported orienting task studies except one, the questions have been presented before the target items. The exception was a study by Craik (1977) in which the words were presented before the questions. In this type of study, there is a holding period between the time the word is presented and the time the question is presented. Craik argued that subjects might use this holding period to carry out more elaborative processing of the word. His results supported this prediction. Recognition performance on words presented with case or rhyme questions improved substantially in relation to performance on words presented with semantic questions, although semantic questions still produced better memory performance than nonsemantic questions. In the Craik study, however, a within-subjects design was used. When the questions are placed after the target items, it is necessary to use a between-subjects design. Otherwise, it can be argued that subjects were processing all the words in the list to the deepest level in order to be prepared for any type of question. With a betweensubjects design, the subjects know what type of question to expect and thus analyze the item only to the required level or depth.

In the following experiments, a between-subjects design was used to compare the effects of different types of orienting questions presented after the stimulus items. Three types of orienting questions were used: those regarding the typeface of the presented word (case questions), those regarding the letters contained in the presented word (letter questions), and those regarding the meaning of the presented word (semantic questions). According to the levels-of-processing model, both the case questions and the letter questions concern orthographic properties of the target items, and consequently, both should produce the same (low) level of memory performance. The semantic questions, on the other hand, should produce a high level of memory performance. If there are qualitative differences among the codes used at the various levels of processing, this pattern should occur whether the questions are placed before or after the target item, although in the questionafter conditions, performance on both orthographic orienting tasks could improve because the subjects may use the holding interval to carry out deeper processing of the word. In order to limit the effects of such processing, there was only a $2-\sec$ period between the presentation of the item and the presentation of the question (in Craik's, 1977, study, there was a 5-sec holding period).

In accordance with a task-demand explanation, however, a different pattern of results should appear. In the question-before conditions, the results should replicate those already obtained in many experiments: Subjects in the orthographic conditions (case-before and letter-before) should perform more poorly than subjects in the semantic condition (semantic-before). In the question-after condition, subjects in the case-after condition should perform at the same level as subjects in 
the case-before condition. In this type of task, the subject is asked questions of the form "Is it in uppercase?" and "Is it in lowercase?". Whether such a question is placed before or after the stimulus item, the subject has to retain only information about the case of the word. In both situations, what is likely to be encoded is the simple fact "uppercase" or "lowercase," not information such as "the word 'cat' was presented in uppercase type." Consequently, when the final test is given, the subject will be able to retrieve only information about typeface, not about the words that were presented.

Similarly, the positioning of the question should not affect performance in the semantic conditions. In a semantic task, the subject is asked questions about the meaning of the item. Whether such a question is placed before or after the stimulus item, the subject must encode the information that "the word presented was "cat"" in order to carry out further analysis regarding its meaning in relation to the presented question. Thus when the final test is given, the subject should be able to retrieve information about the words that were presented.

It is only when the letter questions are asked that we should find a difference between the question-before and question-after conditions. In this type of task, the subject is asked questions of the form "Is there an ' $\mathrm{X}$ ' in it?", where " $X$ " represents a letter that may or may not be contained in the stimulus item. When such a question is placed before the stimulus, the subject need only encode the information that "it contained an ' $\mathrm{X}$ " or "it didn't contain an 'X." When the final test is given, the subject will only be able to retrieve information about individual letters. Therefore, performance on this test should be as poor as that found using other types of orthographic tasks, that is, as in the case-before and case-after conditions. On the other hand, when a letter question is placed after the stimulus item, the subject must encode the information that "the word was "cat" in order to retrieve information about the orthographic features of the word when the question is asked. Thus, when the final test is given, the subject should be able to retrieve information about the words that were presented. This means that memory performance in the letter-after condition should be better than memory performance in the letter-before condition.

\section{EXPERIMENT 1}

\section{Method}

Materials. An acquisition list was made up that consisted of 80 common nouns ranging from four to seven letters in length. A semantic question, a letter question, and a case question were generated for each noun. The semantic questions followed the format used by Craik and Tulving (1975). For instance, the semantic question for the noun "shovel" was "Is it a kind of digging utensil?" and that for the noun "boat" was "Is it a mode of travel?". No two semantic questions were identical to each other. The letter questions followed the format "Is there an ' $X$ ' in it?", in which " $X$ " was replaced by one of the letters in the word. Over the set of 80 corpus items, the letter questions probed all positions in the words approximately the same number of times. There were only two possible case questions, "Is it in uppercase?" and "Is it in lowercase?". In both the semantic question condition and the letter condition, the words in the acquisition list were printed in uppercase type; in the case condition, half of the list was printed in lowercase type and half in uppercase type.

Two versions of the acquisition list were produced. In Version A, 40 of the words in the acquisition list were accompanied by positive-response questions, that is, by the questions that were specifically generated for them and thus required a "true" response. The other 40 words were accompanied by negative-response questions. This was accomplished by interchanging corpus items and the questions designed for them. In Version B of the acquisition list, the true and false sets were reversed, so that all words requiring a "true" response in Version A required a "false" response in Version B and vice versa.

The test list consisted of 80 common nouns, 40 of which had appeared in the acquisition list (the targets) and 40 of which were false distractors. Twenty of the targets were items that had been accompanied by positive-response questions ("yes" targets), and 20 were items that had been accompanied by negativeresponse questions ("no" targets). Given that there were two versions of the acquisition list, the 20 yes targets for Version $A$ would be the 20 no targets for Version B and vice versa. All of the words on the test were printed in uppercase type.

Each stimulus word was presented on a separate slide, as was each question. They were projected onto a screen by a Kodak $850 \mathrm{H}$ slide projector. Attached to the projector was a Gerbrands G1165 shutter, and both were controlled by various components on a BRS logic rack. This apparatus was constructed so that a slide was shown for $2 \mathrm{sec}$, followed by a 2 -sec blank during which the projector changed slides, and then a new slide was shown for $2 \mathrm{sec}$ and the cycle repeated.

Design. There were six encoding conditions, as follows: (1) In the case-before condition, each of the 80 word slides was preceded by a case-classification question slide. The subjects saw the question for $2 \mathrm{sec}$, then a 2-sec blank, and then the word was shown for $2 \mathrm{sec}$, followed by another 2 -sec blank. The subjects were required to circle a "yes" or "no" on an answer sheet during the 2-sec blank that followed the word. (2) The caseafter condition was identical to the case-before condition, except that the question appeared after the word was presented. These subjects saw the word for $2 \mathrm{sec}$, then a 2 -sec blank, and then the question was shown for $2 \mathrm{sec}$, followed by another 2 -sec blank. They were required to circle a "yes" or a "no" on an answer sheet during the 2-sec blank that followed the question. (3) The letter-before condition was identical to the case-before condition, except that letter-classification questions were used instead of case-classification questions. (4) The letter-after condition was identical to the case-after condition, except that letterclassification questions were used instead of case-classification questions. (5) The semantic-before condition was identical to the case-before condition, except that semantic questions were used instead of case-classification questions. (6) The semanticafter condition was identical to the case-after condition, except that semantic questions were used instead of case-classification questions.

In all six conditions, the words were presented in the same order, with positive- and negative-response questions randomly interspersed in the list.

Procedure. The subjects were told that they would see 80 words and 80 questions about these words. Specific instructions appropriate to each condition were given concerning the type of questions that would be asked, and a yes target and a no target were presented using the word "cat" as a demonstration. After all the subjects indicated that they understood the instructions, the acquisition list was presented.

Following this presentation, the subjects were given a $5-\mathrm{min}$ 
interpolated task that involved solving spatial problems. Then they were given the 80 -item test. They were told to circle "yes" if the word in the test had appeared previously as part of the acquisition list and to circle "no" if the word had not been part of the list. The test items also appeared on slides, and each slide was numbered to ensure that subjects would coordinate their answers correctly with the answer sheet. Bach test item was presented for $2 \mathrm{sec}$ with a 2 -sec blank between items.

Subjects. The subjects were chosen randomly from a pool of 1st-year undergraduates attending Memorial University and werc tested in small groups. There were 10 male and 10 female subjects in each of the six encoding conditions. Half the subjects in each encoding condition received Version $\mathrm{A}$ of the acquisition list, and the other half received Version $\mathrm{B}$.

\section{Results and Discussion}

Table 1 shows the mean proportion of correct responses obtained in each orienting condition on the yes targets, no targets, and distractor items. The total average test scores are also presented in the table, so that the six orienting conditions can be compared in overall performance. In the analyses performed on these data, $\mathrm{p}<.05$ was chosen as the level of significance.

The individual scores obtained by subjects were first compared in a 2 (before, after) by 3 (case, letter, semantic) by 2 (targets, distractors) analysis of variance. Three significant effects were obtained from this analysis: There was a difference due to question position $[\mathrm{F}(1,114)=36.25, \mathrm{MSe}=12.52]$, there was a difference among orienting conditions $[F(2,114)=127.29, \mathrm{MSe}=$ $12.52]$, and there was an interaction between question position and orienting conditions $[\mathrm{F}(2,114)=11.17$. $\mathrm{MSe}=12.52]$. The significant difference between question positions appeared to be mainly due to the difference between the letter-before and letter-after conditions. Subjects in the letter-after condition performed much better than those in the letter-before condition. In the other two orienting conditions, performance in the question-after position was only slightly better than performance in the question-before position. The significant difference among orienting conditions was due to the fact that when both positions were combined, subjects given semantic questions performed better than those given letter questions, who in turn performed better than those given case questions. The significant interaction between question position and

Table 1

The Mean Proportion of Test Items Correctly Identified in Experiment 1

\begin{tabular}{lcccc}
\hline & \multicolumn{4}{c}{ Test Scores } \\
\cline { 2 - 5 } \multicolumn{1}{c}{$\begin{array}{c}\text { Orienting } \\
\text { Condition }\end{array}$} & Total & Yes & Norgets & Norgets \\
Targ- & $\begin{array}{c}\text { Dis- } \\
\text { tractors }\end{array}$ \\
\hline Case-Before & .66 & .68 & .64 & .66 \\
Case-After & .68 & .63 & .67 & .70 \\
Letter-Before & .68 & .64 & .60 & .73 \\
Letter-After & .82 & .83 & .81 & .81 \\
Semantic-Before & .88 & .92 & .81 & .89 \\
Semantic-After & .90 & .94 & .85 & .91 \\
\hline
\end{tabular}

orienting conditions reflects the fact that question position had a much greater effect on performance in the letter orienting condition than in the other two orienting conditions.

As there were no significant effects caused by differences in responses to targets and distractors, the six orienting conditions were compared on a NewmanKeuls test using a mean-square error term obtained from a 2 (before, after) by 3 (case, letter, semantic) analysis of variance. The Newman-Keuls comparison found no significant differences among the case-before, case-after, and letter-before groups, but all three of these orienting conditions were significantly poorer than the letter-after, semantic-before, and semantic-after groups. Also, the two semantic groups were significantly better than the letter-after group. This pattern of results was the one predicted by the task-demand explanation. When the question was placed before the target item, subjects in both orthographic orienting conditions performed at the same low level, whereas subjects in the semantic orienting condition performed at a high level on the recognition test. When the question was placed after the target item, performance improved significantly only in the letter orienting condition. It was only in this condition that the task demands changed in response to the question position. When placed before the target item, the letter question did not encourage subjects to encode the target item as a word unit; when placed after the target item, the letter question required subjects to first encode the target item as a word unit.

It is interesting to compare the results obtained in this experiment with those obtained by Craik (1977). Craik reported that subjects given case orienting questions greatly improved in test performance in the questionafter condition. However, in Craik's experiment, subjects did not know what type of question would follow the target, so they had to hold each target in memory for $5 \mathrm{sec}$. In the present experiment, subjects in the caseafter condition did not improve in performance because they did not hold the word in memory until the question was asked; they simply had to remember in what typeface the word was presented.

In Craik's (1977) experiment, subjects in the questionafter condition still performed better on items presented with a semantic question than on items presented with a case or rhyme question. Thus Craik could argue that although the process of holding the word in memory required a deeper analysis of that word, this analysis was not as deep as that required by semantic processing. However, in Craik's study, the difference between the semantic condition and the two others was more pronounced on yes targets. This result was also obtained in Experiment 1. Subjects in the letter-after condition performed almost as well as subjects in the two semantic conditions on the no targets, but they were poorer on the yes targets. This suggests that it is possible that recognition test performance in the letter-after condition 
was significantly poorer than performance in the two semantic conditions only because subjects in the semantic conditions formed elaborated traces to positiveresponse questions.

\section{EXPERIMENT 2}

Craik and Tulving (1975) have suggested that words presented with a positive-response question are better remembered than those presented with a negativeresponse question because the positive-response question produces more elaborated processing of the target word. For example, consider an experimental situation in which the list item is "cat" and the test question is "Is it a domestic animal?". These two items can be integrated to form the coherent idea "A cat is a domestic animal." On the other hand, if "cat" were presented with the test question "Is it an article of furniture?", integration of the question and target word into an elaborated trace would be unlikely. The greater the number of attributes encoded into the trace, the greater the probability that the trace will be retained.

Craik and Tulving (1975) argue that for trace elaboration to occur, the encoded attributes not only must be congruent with the target word but also must provide a more distinctive description of this target. Thus, whereas positive-response semantic questions produce elaborated traces, positive-response orthographic questions do not. If the list item is "cat" and the question is "Does it contain the letter 'c'?", the integrated idea formed from these two items is that "Cat contains the letter "c." Given that so many other words contain the letter "c," the integrated trace does not provide a more distinctive description of the target than a trace containing only the target word. Thus, positive-response questions will produce better memory performance in a semantic orienting condition but not in an orthographic orienting condition.

In Experiment 1, the Newman-Keuls analysis showed that the subjects in the letter-after condition were significantly poorer than the subjects in the two semantic conditions. This difference was more pronounced on the yes targets than on the no targets, although performance on the distractors was poorer in the letterafter condition as well. Performance on distractors, however, might have been affected by the subjects' ability to identify targets.

Experiment 2 was designed to test whether the difference between the letter-after condition and the semantic-after condition would be eliminated if the elaboration effect of positive-response questions was eliminated. In this experiment, subjects saw the same acquisition corpus as subjects in Experiment 1, but the test contained only those target items that were presented with negative-response questions. Without yes targets in the test, elaboration would not be a factor in test memory performance. The only factor that could produce a difference between semantic and orthographic tasks would be the level to which the target word was processed.

\section{Method}

Materials. The acquisition lists and apparatus were identical to those used in Experiment 1. Two new test lists were created, both containing as target items only those words that had been accompanied by negative-response encoding questions. As per Experiment 1, the test list consisted of 40 targets and 40 distractors.

Procedure. There were three encoding conditions in Experiment 2, a semantic-after condition, a letter-after condition, and a case-after condition. These were identical to those used in Experiment 1. Also, the presentation and testing procedures were identical to those employed in Experiment 1.

Subjects. The subjects were chosen randomly from a pool of 1st-year undergraduates attending Memorial University and were tested in small groups. None had taken part in Experiment 1 . There were 10 male and 10 female subjects in each of the three encoding conditions. Half of the subjects in each encoding condition received Version $A$ of the acquisition and test lists, and the other half received Version $\mathrm{B}$.

\section{Results and Discussion}

The mean proportion of targets and distractors correctly identified is shown in Table 2 . Subjects in the semantic-after and letter-after conditions performed at approximately the same level, and both groups were considerably better than subjects in the case-after condition. A 3 (case, letter, semantic) by 2 (targets, distractors) analysis of variance found only a significant difference between orienting conditions $[\mathrm{F}(2,57)=$ $7.35, \mathrm{MSe}=17.55]$. As there were no significant effects due to differences in responses to targets and distractors, the three orienting conditions were compared using a mean-square error term obtained from an analysis of variance comparing the three conditions. This NewmanKeuls analysis showed a significant difference between the case-after and letter-after conditions and between the case-after and semantic-after conditions, but no significant difference between the letter-after and semantic-after conditions.

The results of Experiment 2 supported the hypothesis that the difference between the letter-after and

Table 2

The Mean Proportion of Test Items Correctly Identified in Experiment 2 and in the Replication Experiment

\begin{tabular}{lccc}
\hline & \multicolumn{3}{c}{ Test Scores } \\
\cline { 2 - 4 } Orienting Condition & Total & Targets & $\begin{array}{c}\text { Dis- } \\
\text { tractors }\end{array}$ \\
\hline & \multicolumn{3}{c}{ Experiment 2} \\
Case-After & .64 & .65 & .63 \\
Letter-After & .71 & .71 & .71 \\
Semantic-After & .73 & .75 & .70 \\
& & Replication \\
Letter-After & .74 & .78 & .70 \\
Semantic-After & .71 & .70 & .72 \\
\hline
\end{tabular}


semantic-after conditions would disappear once the effect of elaboration to positive-response questions was eliminated. However, the identification of target items was slightly, if not significantly, better in the semanticafter condition than in the letter-after condition. Therefore, the experiment was replicated, comparing only the letter-after and semantic-after conditions, with 12 subjects in each condition. The results of this replication are also shown in Table 2. In this replication, subjects in the letter-after condition performed slightly better than subjects in the semantic-after condition, and again, there were no significant effects between these two orienting conditions.

\section{GENERAL DISCUSSION}

Although it is well known that semantic orienting tasks produce better retention of target items than nonsemantic orienting tasks, the reason for this difference has not been clearly delineated. The most commonly accepted explanation for the orienting task phenomenon, the levels-of-processing model, argues that semantic judgments produce a deeper analysis of the target items than do nonsemantic judgments. This model has, however, failed to provide a definition of depth that is independent of the nature of the tasks used to measure depth (cf. Baddeley, 1978). In recent years, a wide variety of researchers studying a wide variety of phenomena have used the concept of "deeper processing" as an explanatory variable. In view of its popularity, we should provide as clear a definition as possible of exactly what mental activities produce deeper processing.

The experiments in this paper were designed to test how well the task-demand hypothesis could explain the orienting task phenomenon. The task-demand hypothesis assumes that there is only one kind of memory code, a semantic code, which differs in content according to the demands of the task at hand (cf. Morris et al., 1977). A task that requires subjects to process information about the referential aspects of a target item will result in information's being retained about the meaning of this item. Such a task also requires that the target item be encoded as an identifiable unit before processing of its semantic characteristics are carried out. On the other hand, a task that requires subjects to process information about the orthographic aspects of the target item will result in information's being retained about these orthographic characteristics. Such a task may not require that the target item be encoded as an identifiable unit. To decide whether a given word is printed in uppercase type or lowercase type, a subject need process only one letter of the word and encode the information "uppercase" or "lowercase." To decide whether the word contains a specific letter, a subject is encouraged to process each letter separately rather than encode the word itself as an entity. If the only information encoded was the fact that one of the presented items contained a " $t$," the encoder would be unable to decide whether or not "cat" had been presented.

The task-demand hypothesis can be reformulated in terms of the encoding-specificity principle (cf. Tulving \& Thomson, 1973). The encoding-specificity principle states that successful retrieval occurs when the operations performed during retrieval closely match the operations performed when the trace was first encoded. Thus, if the orienting questions ask the subject to encode information about rhyming characteristics of the target items, it will be easier to retrieve this information in response to a rhyming test than in response to a semantic test. Results supporting the encoding-specificity principle were obtained by both Fisher and Craik (1977) and Morris et al. (1977). Fisher and Craik, however, argued that the encoding-specificity principle could not completely explain the orienting task results because performance in the nonsemantic condition was not equivalent to performance in the semantic condition even when the appropriate retrieval mode was used. Fisher and Craik stated that it was still necessary to retain the notion of processing depth to explain the orienting task findings. Consequently, we are still left with the problem of defining the exact nature of "processing depth."

It is possible that differences in processing depth occur because the memory trace produced in response to a semantic orienting task is qualitatively different from that produced in response to a nonsemantic orienting task. In other words, it may be that semantic information is stored in a semantic memory code and nonsemantic information is stored in a nonsemantic memory code. For example, orthographic information could be represented as an image of the letters of the target word, phonemic information, as an image of the sounds of the word. If such nonsemantic images are retained, this information should be retrievable from the memory trace. Thus subjects should be able to retrieve information about the orthographic features of the presented targets independent of whether or not they encoded semantic information about these targets.

The results of the two experiments in this paper did not support the predictions of a different-memory-codes hypothesis. Subjects in the letter-after condition needed only to retain and analyze the orthographic features of the target items, whereas subjects in the semanticafter condition needed to retain and analyze the semantic features of these targets, yet there were no differences between these two conditions on the retention test presented in Experiment 2. On the other hand, subjects in the case-after condition, like those in the letter-after condition, also needed to retain orthographic information about the targets, yet their performance on the retention test was much poorer than subjects in the letter-after condition. These findings are not consistent with the concept of qualitatively different memory codes, but they are consistent with the task-demand 
hypothesis. The task demands in the case-after condition required only that information about the typeface of the presented items be retained during the holding period between presentation of the item and presentation of the question. The task demands in the letterafter and semantic-after conditions required that information about the identity of the presented items be retained during the holding period. The difference between the letter-after and semantic-after conditions lay in the nature of the analyzing operations performed in response to the questions asked after the targets were encoded as identifiable items. This analyzing difference did not produce superior memory retention in the semantic-after condition once the factor of trace elaboration was removed. Thus semantic analyzing operations do not result in better memory retention per se. This means that a major component of processing depth is the degree to which the task encourages the subjects to encode the targets as identifiable entities. Semantic orienting tasks would usually produce better memory performance because such tasks are more likely to induce a subject to encode the target items as identifiable units. Obviously, even with an orthographic task, there is some possibility of encoding target items as identifiable units, as performance on memory tests presented after such orienting is usually above chance level. The probability of encoding target items as identifiable units should vary in accordance to the particular task employed and in response to the method used in presenting the task.

Fisher and Craik (1977) argued that encoding specificity (or the task-demand hypothesis) could not offer a sufficient explanation for the orienting task phenomenon. They did not, however, specify what factor or factors other than encoding specificity were necessary components of processing depth. The findings obtained from the experiments in the present paper support the general position taken by Fisher and Craik. The taskdemand hypothesis did not offer a sufficient explanation for the experimental results. To explain all of the findings, it was necessary to include the concept of trace elaboration.

According to Craik and Tulving (1975), an elaborated trace is one containing the representation of the target item plus additional distinctive information about this target. When positive-response questions are presented with the target, the information in the question is incorporated into the trace containing the representation of the target. This produces an elaborated trace, and the more elaborated or complex the trace, the easier it is to retrieve. Negative-response questions do not produce elaborated traces because the target and the question information cannot be represented as a congruous, integrated unit. Also, positive-response questions about the orthographic features of the target do not produce elaborated traces because the information in the ques- tion does not provide distinctive or unique information about the target. Therefore, trace elaboration would have a much greater effect on performance in a semantic orienting condition than in an orthographic orienting condition. This was precisely the result found in the present study. In Experiment 1, both yes and no targets were presented in the recognition test and performance in the semantic-after condition was significantly better than performance in the letter-after condition. In Experiment 2, only no targets were presented in the recognition test, consequently eliminating any possible effect of trace elaboration on performance, and there were no significant differences between the semanticafter and letter-after conditions.

Thus the results obtained in the present experiment suggest that the orienting task phenomenon can be explained in terms of two processing components. The task-demand component specifies that retention level is determined by the degree to which the orienting task encourages subjects to process the target items as identifiable units. The higher the probability of encoding targets as identifiable units, the higher the level of retention. The trace elaboration component specifies that retention level is also determined by the degree to which the orienting questions encourage subjects to form elaborated traces. The greater the amount of distinctive information processed about the target items, the easier these items will be to retrieve. If an investigator wants to eliminate the trace elaboration factor from the study, yes targets should be eliminated from the retention test measure. By manipulating the type of target used in the memory test, we can discover the degree to which the two components of processing depth contribute to a particular experimental effect.

\section{REFERENCES}

BAdDeley, A. D. The trouble with levels: A reexamination of Craik and Lockhart's framework for memory research. Psychological Review, 1978, 85, 139-152.

Craik, F. I. Depth of processing in recall and recognition. In S. Dornic (Ed.), Attention and performance VI. Hillsdale, N.J: Erlbaum, 1977.

Craik, F. I., \& Lockhart, R. S. Levels of processing: A framework for memory research. Journal of Verbal Learning and Verbal Behavior, 1972, 11, 671-684.

Craik, F. I., \& Tulvina, E. Depth of processing and the retention of words in episodic memory. Journal of Experimental Psychology: General, 1975, 104, 268-294.

Fisher, A. M., \& Craix, F. I. The interaction between encoding and retrieval operations in cued recall. Journal of Experimental Psychology: Human Learning and Memory, 1977, 3, 701-711.

HyDE, T. S. Differential effects of effort and type of orienting task on recall and organization of highly associated words. Journal of Experimental Psychology, 1973, 97, 111-113.

Hyde, T. S., \& Jenkins, J. J. The differential effects of incidental tasks on the organization of recall of a list of highly associated words. Journal of Experimental Psychology, 1969, $82,472-481$.

Hyde, T. S., \& Jenkins, J. J. Recall for words as a function 
of semantic, graphic or syntactic orienting tasks. Journal of Verbal Learning and Verbal Behavior, 1973, 12, 471-480.

JACOBY, L. L., \& Craik, F. I. M. Effects of elaboration of processing at encoding and retrieval: Trace distinctiveness and recovery of initial context. In L. S. Cermak \& F. I. M. Craik (Eds.), Human memory: A cognitive view. Hillsdale, N.J: Erlbaum, 1978.

Johnston, C. D., \& Jenkins, J. J. Two more incidental tasks that differentially affect associative clustering in recall. Journal of Experimental Psychology, 1971, 89, 92-95.

Mosser, S. D. Effect of questions on prose unitization. Journal of Experimental Psychology: Human Learning and Memory, 1978, 4, 290-303.

Morris, C. D., Branbford, J. D., \& Franks, J. J. Levels of processing versus transfer appropriate processing. Journal of Verbal Learning and Verbal Behavior, 1977, 16, 519-533.

Rosenbero, S., \& Schiller, W. J. Semantic coding and inci- dental sentence recall. Journal of Experimental Psychology, 1971, 90, 345-346.

Schulman, A. I. Recognition memory for targets from a scanned list. British Journal of Psychology, 1971, 62, 335-346.

Schulman, A. I. Memory for words recently classified. Memory \& Cognition, 1974, 2, 47-52.

Tulving, E., \& Thomson, D. M. Encoding specificity and retrieval processes in episodic memory. Psychological Review, $1973,80,252-272$.

WALBH, D. A., \& JEnKing, J. J. Effects of orienting tasks on free recall in incidental learning: "Difficulty," "effort" and "process" explanations. Journal of Verbal Learning and Verbal Behavior, 1973, 12, 481-488.

(Received for publication February 15, 1982; accepted October 4,1982 .) 